\title{
Dante sulle colline
}

\author{
GiovanNA IOLI \\ Università degli Studi di Torino \\ giovanna.ioli@tin.it
}

\section{Riassunto}

Il saggio percorre gran parte della produzione in prosa di Cesare Pavese (Paesi tuoi, Feria d'agosto, La luna e i falò, Il mestiere di vivere) alla ricerca della traccia dantesca nella creazione letteraria dello scrittore italiano. Tanto l'espressività dei migliori testi di Pavese quanto la ricchezza ermeneutica della sua opera sono in parte dovute alla forza simbolica ed allegorica di alcuni dei suoi più memorabili passaggi tragici, la cui prima «fonte» originaria italiana ci conduce fino a Dante Alighieri.

Parole chiave: Dante, simboli, allegorie, Pavese.

\section{Dante on the hills}

\begin{abstract}
The essay deals with most of the prose of Cesare Pavese (Paesi tuoi, Feria d'agosto, La luna e i falo, Il mestiere di vivere) trying to find the dantian trace in the Italian writer's literature. Both the expressiveness of the best texts of Pavese and the hermeneutic richness of his work are in part indebted to the allegoric and symbolic tragic passages, whose original «source» leads to Dante Alighieri.
\end{abstract}

Key words: Dante, symbol, allegory, Pavese.

Ioli, Giovanna. 2011. Dante sulle colline. Cuadernos de Filología Italiana, $\mathrm{n}^{\mathrm{o}}$ extraordinario: págs. 209-218.

La cronaca dei rapporti testuali tra Dante e Pavese non può prescindere dallo scarto quantitativo che si riscontra tra le opere destinate alla pubblicazione (poesia e narrativa) e gli scritti privati, quali il Mestiere di vivere, gli epistolari o i taccuini. Nelle prime, infatti, ci troviamo di fronte a una calcolata reticenza, una «dissimula- 
zione onesta» - come insegnava Torquato Accetto $(1641)^{1}$ - chiamata a sostituire le allusività interne segnate di libresco con immagini argute, «astutissime» (Mutterle 1977: 43-88). ${ }^{2}$ Nei secondi, invece, il riferimento ai modelli è un dichiarato frutto di studi, a cominciare dalle lezioni che avevano forgiato la «Confraternita del D'Azeglio», che «per non perdere le due ore di lezione dantesca di Monti il lunedì pomeriggio» si alzavano dal letto «con trentanove di febbre e l'influenza» (Mila 1977: VI): un'attenzione che continuò anche oltre la soglia dell'esame di maturità, grazie alla «virgiliana» cura di un maestro, convinto che il condurre quei ragazzi alla salvezza attraverso i libri avrebbe avuto un seguito. Augusto Monti, che si trasfigurava leggendo la Commedia, aveva certamente trovato un terreno dissodato nello studente che si era già cimentato, ancor prima d'incontrarlo, con un poemetto in terzine dantesche su trama salgariana, dove comparivano spie lessicali e ritmiche (quali «bronchi», «quelli: d'elli»), segnalate da Lorenzo Mondo anche nell'ultimo libro dedicato a «quell'antico ragazzo» (Mondo 2006: 13). ${ }^{3}$ Le lettere inviate agli amici di quegli anni o le annotazioni autobiografiche raccolte nei taccuini confermano che quelle lezioni avevano prodotto una sorta di furore creativo, che in Pavese alternava momenti di impotenza di fronte a tale modello, ma anche «superbia» - come scrive il 21 febbraio del 1925 - «che la simile non l'aveva Dante. Ma con ciò non voglio pormi sopra Dante: vedo ora molto più chiaro di una volta, quanto questo grande sia alto» (Lettere I 1966: 6).

Nella corrispondenza con Mario Sturani, che talvolta ricalca i toni di tenzoni stilnovistiche ed evoca angelicate assenze, l'intento di mettere a frutto quelle lezioni è evidente e lo testimonia in particolare il sonetto del 1923, Messer Sturani, puoi cessar lo canto e la coeva «poesia della loda», Un sogno (Mario Sturani 1990: 16). ${ }^{4}$ Pavese parla apertamente di questa vena con Tullio Pinelli il 12 ottobre 1926, dicendo di essere, «ad onta di tutti i miei ruggiti e le mie smorfie da ribelle e da scettico, l'antico trovatore di quelle corti d'amore che sai».

Guido, i` vorrei che tu e Lapo ed io / fossimo presi per incantamento...Tu non sai per quanto tempo ho respirato nella Vita Nuova così corrotto di corpo e d'anima come sono. Sturani, quella specie di mio fratello maggiore, ed io amiamo talvolta discorrere delle nostre due arti poetiche (?!?!?!?) come di un dolce stil novo (Pavese 1974: 13).

Sono anni, dunque, in cui Pavese si accende di vigore leggendo la Vita Nova, «vorrebbe puntare alle cose grandi, avere un grande destino, scrivere "qualcosa che

\footnotetext{
1 Nel presentare il suo trattato l'autore diceva di aver scelto «di rappresentar il serpente e la colomba insieme, con intenzion di raddolcir il veleno dell'uno e custodir il candor dell'altra (come sta espresso in quelle divine parole: "Estote prudentes sicut serpentes, et simplices sicut columbae")».

2 L'aggettivo è un esempio del wit shakespeariano studiato ampiamente da Anco Marzio Mutterle (1977).

3 Lorenzo Mondo ne aveva dato conto già (1964: 3-21).

${ }^{4}$ Le due poesie datate 20/27 marzo e 2 maggio 1923 sono state pubblicate nelle edizioni fuori commercio in occasione del quarantennale della morte (Pavese 1898: 5-7).
} 
duri in eterno" (Lajolo 1960: 88), mettendo a frutto la «tecnica fortissima», «formidabile», la «sintesi di azione», la «vivacità» e il «colore» delle poesie giovanili di Dante e non «per pochezza d'ingegno», aggiunge, ma per meglio esprimere «il fondo intimo della mia anima». Nel rispondere a Mario Sturani, che usa proprio quest'argomento per far leva sul timido corrispondente, nella convinzione che quei testi potessero trasformarsi in un modello, Pavese annuncia in tono programmatico il suo proposito:

Conto un giorno o l'altro, di andare a ispirarmi, ad accendermi (fuochi di paglia) dinanzi alla Vita nuova. Le darò uno sguardo, cercando di non alterarlo col mio pensiero (Pavese 1966a: 4).

In questo suo apprendistato stilnovistico alterna momenti di sfiducia con altri di esaltazione, «un po' è Dante, un po' è fesso, sempre in tema, desideroso di superar tutti tanto che supera nessuno e muore nelle sue misere applicazioni di volontà», come il suo alter ego, il giovane indeciso dell'abbozzo di romanzo Lotte di giovani (1993: 185). Proprio la volontà «superba», tuttavia, lo spingerà a «studiare, studiare», «colmare l'anima di poesia altrui», di quelle opere che - lo dice a Monti in una lettera dell'agosto 1926 - «sono il riassunto di secoli conservati appunto viventi» (Pavese 1974: 8).

La tormentata ambizione giovanile si trasformerà così in un metodo indirizzato fin dagli esordi verso la preminenza di una mediazione letteraria che rifiuta la mimetica dei «poemi sporchi», come si legge in una lettera del '32, quelli contaminati da un modello statico, sperimentando, invece, un realismo congegnato su due piani, capaci di fondere consapevolezza critica ed esperienza vissuta, dissolvendo così nel nuovo testo sia il reale biografico sia la tradizione alta ${ }^{5}$. Non si tratta solo di un incanto giovanile, tanto che Dante ritorna, affiancato a Shakespeare, negli appunti degli ultimi giorni di confino, dove i suoi antiqui prediletti si sono ormai interiorizzati come «consorti», «potenti, favolosi costruttori che si deliziano del particolare, sentito fino allo svolazzo, che respirano tutta la vita / a regolari e perfetti respiri quotidiani». Il 23 febbraio 1936 Pavese li definisce «veggenti benparlanti» in cui lungimiranza ed elequenza si assommano, «tutti cose, tranquilli e impassibili suscitatori della varietà, i sornioni dell'esperienza, che la sfaccettano in figure come a gioco, finendo a sostituirla, astutissimi» (Pavese 2000a: 29).

Dante, Shakespeare, seguiti da Omero, attraversano il Mestiere di vivere ed entrano a far parte di un ragionamento che dal 1939 in poi avrà come scorta fida anche Platone, il teatro greco e Dostojevskij. Il 9 marzo del 1948, solo due anni prima della fatidica soglia, lo ribadiva quasi a suggello di un'opera che aveva intinto la sua penna anche in quell'inchiostro: «I quattro più grossi mondi -complessi e inesauribili, ambigui, moderni- sono Platone, Dante, Shakespeare e Dostojevskij» (Pavese 2000a: 348).

\footnotetext{
${ }^{5}$ Una forma d'arte che «porta l'uomo più d'ogni altra cosa vicino alla divinità» e «permette di creare esseri vivi», lo spinge a inseguire un'idea condotta per linee esterne (Pavese 1993: 94).
} 
Il riscontro di questa lunga fedeltà sul piano privato, ben nota agli studiosi di Pavese, non sembra rispettare le stesse misure volumetriche nelle opere destinate alla pubblicazione, al punto di scoraggiare un discorso sulla lezione dantesca, che non compare nella pur monumentale bibliografia critica che lo riguarda (Mesiano 2007). C'è, infatti, una vera e propria resistenza testuale, come se l'esempio dei «consorti» fosse presente in una consapevole assenza di vocabolarizzazione, nella convinzione che la lingua poetica di Dante «non ha potuto, né potrà mai servire di modello a composizioni in prosa» (Pavese 2000a: 213). L'apparente distanza dalla tecnica di riuso lessicale della tradizione illustre era, dunque, dichiarata fin dalle origini, puntando piuttosto verso una «poesia di situazione» (Pavese 2000a: 18) e un realismo simbolico che incastona negli eventi descritti elementi che li trascendono, riflettendo il pavesiano «bisogno di 'credere avvenuto' ciò che raccontiamo» (Pavese 2000a:163). Questo giustifica la carenza di citazioni testuali riconoscibili come tali, sparse qua e là come briciole cadute da un piatto troppo pieno o, forse, argutamente mirate per mettere in evidenza non tanto il prestito lessicale, ma una «situazione» stilistica in grado di appagare la sua vocazione all'unità dell'opera, costruita come rappresentazione narrativa di un simbolo, evocando per speculum e per enigmi anche altre poetiche. Il tema del viaggio lo testimonia, diventando l'esempio principe, lo strumento che permette di assommare esperienze libresche in cui Dante, Whitman o Melville si fondono per costruire la «situazione» di una nuova avventura individuale. Non è, dunque, il calco della lingua dei modelli a essere scelta come spia dell'astuto riuso della tradizione, ma una sua personale interpretazione, chiamata in causa come forgia di un nuovo ordine che ha assorbito come un buco nero anche le testimonianze capitali della storia letteraria. "Artisti come Dante (lo Stilnovo), Stendhal e Baudelaire sono creatori di situazioni stilistiche», annota il 1 gennaio 1940 (Pavese 2000a: 169): "sono gente che non cade mai nella bella frase, perché concepiscono la frase come creatrice di situazioni». La lingua della tradizione, insomma, è un ricordo intangibile già consegnato alla memoria storica, uno stereotipo sacro che lo spinge, questo sì, a crearne altri, per costruire una nuova «storia letteraria vista da un poeta come storia della propria poetica» (Pavese 2000a: 239). La lezione dei «consorti» viene dunque usata come strumento di sperimentazione «realistica» nutrita di antiqui e deve essere riconosciuta come tale per affrancare le sue storie dal sospetto di «un Piedemontese Revival» (Pavese 2000a: 19), anche se le impronte e i riverberi lessicali che segnalano un percorso ancorato al passato, sono continuamente rinnovati «dal tempo e dalla dimenticanza frammessa», fino a farli diventare «misteriosamente» nostri (Pavese 2000a: 283). Lo attestano gli appunti di lingua dello scrittore e alcune varianti che riportano il suo paesaggio nel solco della lezione dantesca. In Feria d'agosto, per esempio, troviamo la parola «torrente» che diventa «gora», «plaga» invece di «distesa», «ficcare gli occhi» al posto di «guardare». Lo stesso si può dire per parole come «costa» e «balzo» del racconto Il mare o «proda» della Luna e i falò e quel «Ci fermammo in co' della vigna» segnalato da Beccaria, che è sì dantesco (in «co' del ponte» di Purgatorio III 128) ma anche dialettale, per far sì «che ogni eco dell'alto prenda nuova linfa e rinforzo dal basso, dal regionale o dal dialettale» (Beccaria 2000: XIX). La presenza dell'exemplum dantesco, insomma, riaffiora nell'ambito di un'idea in grado di testimoniare che anche $\mathrm{i}$ 
«vuoti» hanno in Pavese un diritto di significanza, che emerge dalla consapevolezza di quanto sia «arduo trasformare se stesso in un io dantesco, simbolico, quando i propri problemi sono radicati a un'esperienza così individuale come la città-campagna e tutte le trasfigurazioni giungono soltanto a simboli psicologicamente individuali» (Pavese 2000a: 281).

La sua idea, allora, si avvale di una particolare tecnica espressiva, fondata su un senso continuamente rinnovato del paesaggio, destinato a diventare particolare ovvero «la consacrazione di luoghi unici» cui «si dà un significato assoluto, isolandolo nel mondo» (Pavese 2000a: 57) - e universale, come «il "cammino dell'anima" della mia Divina Commedia» (Pavese 2000: 164): da Paesi tuoi, «natio loco» e non semplicemente strapaese a La luna e i falò, un libro nato, dantescamente, da «una mirabile visione» grazie alla quale - diceva Pavese - «dovrei costruire una mia modesta Divina Commedia». Così scriveva il 17 luglio 1949, solo due mesi prima di cominciare la stesura della Luna e i falò, la chiusura del cerchio (Pavese1966b: 399). Nei due romanzi che segnano l'arco di una vita, Pavese come il Dante purgatoriale, «non si volta mai a contemplare il panorama, per la ragione che non descrive realisticamente un viaggio, ma espone un simbolo dove si ricorre alla scena, al visibile, solo in quanto si riveste di corpo un concetto. Non ha quindi obblighi di rispettare la logica naturalistica del reale» (Pavese 2000a: 163). I «simboli danteschi dice il 4 dicembre 1938 - non sono già la Croce e l'Aquila... ma, per esempio, la nota crepuscolare di tutti gli episodi del Purgatorio che dà e prende significato alle visioni e che esprime il messaggio di un mondo che sfuma gioiosamente» (Pavese 2000a: 142$)^{6}$.

Proprio sulle basi di questo assunto di «situazioni» e di «concetti» è possibile testimoniare il dantismo pavesiano con qualche riscontro testuale, abilmente dissimulato, a cominciare da Paesi tuoi, l'inizio del viaggio, il cammino anche linguistico che assorbe la tradizione illustre neutralizzandola (Beccaria 1989: 75), lasciando filtrare anche una nuova realtà di «parola» antica, perché per lui «la cultura deve cominciare dal contemporaneo e documentario, dal reale, per salire - se è il caso ai classici» (Pavese 2000: 396). È una sorta di principio dei vasi comunicanti, che lascia confluire nel racconto il volgare antico e quello moderno, pronunciando nomi comuni ma immessi in situazioni universali, che escludono la possibilità di «errori fantastici» (Pavese 2000: 22) o di campanile, trasformando tutti i prati, le selve, le colline in luoghi mitici, trascendentali, perché la poesia, quella poesia, «non fa che dare un' esistenza immortale alla vita» e alla letteratura.

Nel viaggio di Paesi tuoi verso le origini, infatti, orrore e malinconia, abiezione e tenerezza, cammino verso la cima di una collina amorosa come una mammella, sono impressi con un ritmo che ricalca il dinamismo del pellegrino; un'ascesa che

\footnotetext{
6 Non è un caso che Pavese citi «la Croce e l'Aquila», perché, secondo una testimonianza di Tullio Pinelli del '79, allude alle «lezioni» di un celebre dantista dei suoi tempi, Don Brizio Casciola, «che interpretava la Divina Commedia secondo la teoria del Valli (l'Aquila e la Croce): una interpretazione dotta e teorica del tutto opposta a quella che ce ne dava a scuola il professor Monti. Naturalmente, ho tutto dimenticato di ciò che don Brizio diceva, mentre le lezioni di Monti hanno formato il mio modo di pensare».
} 
esibisce rare citazioni riconoscibili, ma che usa la lezione dantesca per diventare simbolo di un viaggio verso il riscatto, la fine della pena.

«Stasera mangerai la gallina con tuo padre, gli dico guardando la strada». Così esordisce Pavese nel romanzo in cui Berto è la guida e Talino il disperso che cerca protezione e, forse, spera in una redenzione. «Lui mi veniva dietro», dice Berto, in un'ascesa apparentemente così poco metafisica da scoraggiare ogni interpretazione che abbia a che fare con Dante. Eppure ci rendiamo conto che dal linguaggio comune continuano a filtrare tasselli per costruire una «situazione» che è quasi un calco parodico e shakeapearianamente arguto, dell'inizio della Commedia. «Lui mi veniva dietro», allora, comprende anche «io dietro mi mossi» di Inf. XVIII 21 o altri luoghi come «tirandosi me dietro sen giva» di Purg. XXXI 95, ovvero parole riferite da Dante per ben altra guida. Talino era timoroso al punto, continua Berto, che «mi stava attaccato come se il carrettino dei gelati che passava tutta corsa minacciasse noi due pedoni». E poi aggiunge: «Né io né lui ci rivoltammo a guardare le Carceri». ${ }^{7}$ Se la situazione evocata è proprio quella dantesca, allora, è anche ragionevole ipotizzare che nel timore di Talino si rifletta anche la paura che coglie Dante durante il suo viaggio («mi strinsi al poeta per sospetto», Inf. IX 51; «m'accostai, / temendo, al duca mio», X 30-31; «mi volsi intorno, e stretto m'accostai, / tutto gelato, a le fidate spalle» Purg. VIII 41-42), già manifesta nella selva oscura, tanto da meritarsi il rimprovero di Virgilio («ogne viltà convien che qui sia morta», Inf. III 15), turbato nel vedere la sua anima «da viltade offesa» (Inf. II 45). Così pure l'immagine del carcere non è semplicemente un dato topografico, ma l'assimilazione del «doloroso carcere» (Inf. XXXIII 56): il «cieco / carcere» infernale (Inf. X 59) che i due pellegrini stanno attraversando.

Nel panorama che si apre agli occhi di Berto e Talino, inoltre, «le piante spesse del viale», potrebbero ricalcare quel «cammino alto e silvestro» di Inf. II 142, mentre quel «gran caldo», «il colore del caldo», il «cielo caldo», «il rumore del sole come fosse un incendio» (pp. 44, 52, 91), richiamano una temperatura estrema, infernale, come «ne le tenebre etterne, in caldo e 'n gelo» di Inf. III 87 e le altre di Purg. III 31, dove si sta «a sofferir tormenti, caldi e geli».

«Non c'è nessuno in queste strade» che i due amici percorrono e come nella «piaggia diserta» anche loro vanno «avanti come i buoi senza sapere dove», che potrebbe serbare un'eco di quel «di pari, come buoi che vanno a giogo» di Purg. XII, 1.

Sono pochi indizi, è vero, ma sparsi in una trama d'immagini ripetute e rinnovate, assimilate al punto da riflettere una situazione campestre da selva oscura e un itinerario nel reale eretto a simbolo, ma svincolato dal naturalismo e dal realismo. Il paesaggio autobiografico, $\mathrm{i}$ «paesi tuoi», insomma, sono il «natio loco», il marchio di un linguaggio che sfugge di poco all'allegoria e che Pavese definirà nel suo diario un legame fantastico «che tende una trama sotto il discorso ... una realtà segreta che affiora ... Non più simbolo allegorico, ma simbolo immaginoso - un mezzo

${ }^{7}$ Il «carcere» chiama in causa le «invisibili pareti di una cella» del romanzo Il carcere, dove è evidente che l'immagine ossessiva della prigionia è figura della condizione esistenziale. 
di più per esprimere la «fantasia»»». Parallelo di questo mezzo, aggiunge, non è tanto l'allegoria quanto l'immagine dantesca. ... Il XXIII del Paradiso può suggerirci» (Pavese 2000a: 165). Suggerirci cosa? Il canto da lui chiamato in causa è quello in cui «tutti quei fenomeni di luce ... dicono la realtà luminosa del luogo»e anche la sua realtà segreta di «foce di tutte le cose create». Forse è proprio quella foce di poesia segreta a trasformare anche il racconto di Pavese in qualcosa che travalica il reale, che va osservato con occhi abituati a quel fulgore. Lungo il cammino di Paesi tuoi, la luce e gli occhi che guardano la realtà trasfigurata in simbolo si richiamano in tutto il romanzo per cenni di castella, costruendo un aperto contrasto tra la speranza di lavorare nella «fabbrica della luce» e gli occhi di chi - «anime perse» - non ha ancora raggiunto la salvezza. Sono occhi animaleschi, di bue, di merlo, storti, ammiccanti, spaventati, che girano «da tutte le parti come si gira una paglia tra $\mathrm{i}$ denti». Girano come quelli di Caronte che li «avea di fiamme rote».

L'inchiostro di Dante fermenta con la stessa formula anche nel componimento che apre la raccolta Lavorare stanca, considerato per esplicita dichiarazione la sua selva oscura (Pavese 2000a: 12) in cui «per la prima volta» cominciò a «costruire una persona spirituale» (Pavese 2000a: 8). Nel saggio posto in appendice all'edizione definitiva delle Poesie, Pavese spiegava ancora una volta che il suo modo di raccontare immagini doveva evocare «una realtà non naturalistica ma simbolica», non un'autobiografia ma un giudizio. "Come succede insomma nella Divina Commedia - (bisognava arrivarci) -, avvertendo che il tuo simbolo vorrà corrispondere non all'allegoria ma all'immagine dantesca» (Pavese 1998: 118).

I Mari del Sud, scritta nel settembre 1930 è intessuta in una trama che evoca Dante senza mai citarlo esplicitamente, già con l'intento di cercare «una superba avventura individuale, in cerca del sublime anche nell'umile», un'esigenza soprattutto linguistica, che lo condurrà «alla fine del suo itinerario di scrittore al "volgare" illustre, al "piemontese" illustre della Luna e i falò» (Beccaria 1989: 80 e 71).

Il primo verso, «Camminavo una sera sul fianco di un colle», segnala ancora una volta l'inizio di un viaggio che richiama ben altro «cammin di nostra vita» (Inf. I 3), ma anche una topografia che mostra il «fianco di un colle» per Pavese e «la costa di traverso» di Purg. V 22, l' «oscura costa» di Inf. II 40 e il celebre «colle» di Inf. I 13 per Dante.

Lo stesso verbo dinamico ritorna nei versi successivi e nella seconda strofe è accompagnato da una di quelle briciole spia che rimandano a un modello ormai incastonato nel suo paesaggio. Il cugino che lo accompagna, infatti, «cammina per l'erta», come Dante «al cominciar de l'erta» del colle illuminato dal sole di Inf. I 31; VIII 128.

Nella sesta strofe, invece, nei versi che recitano: «Camminiamo da più di mezz'ora. La vetta è vicina / sempre aumenta d'intorno il frusciare e il fischiare del vento», ci si imbatte ancora nella «situazione» ormai impostata in odore di Dante, tale da stimolare anche il ricordo del «suon della foresta» di Purg. XXVIII 85 e il fruscio delle fronde sulla vetta del monte, dove il tema del passo ha la sua consacrazione allegorica.

Tra le righe, inoltre, aleggiano altri possibili rimandi, il silenzio soprattutto, 
In silenzio. Nell'ombra del tardo crepuscolo mio cugino è un gigante vestito di bianco, che si muove pacato, abbronzato nel volto, taciturno. Tacere è la nostra virtù.

L'insistenza di parole quali «silenzio», «pacato», «taciturno», «tacere», irrompono come tema proprio della campagna, in aperto contrasto con la quarta strofe, là dove dice che

La città mi ha insegnato infinite paure:

una folla, una strada mi han fatto tremare, a migliaia sul gran scalpiccio.

Il silenzio è la prima cosa che Dante rileva nell'incontro con Virgilio («dinanzi a li occhi mi si fu offerto / chi per lungo silenzio parea fioco», Inf. I 62-63), in pieno contrasto con il fragore della «città dolente» (Inf. III 1) «che 'ntrono accoglie d'infiniti guai» (Inf. IV 9), tali da far «tremar le vene e i polsi» del poeta (Inf. I 90). Altri arguti intarsi di situazioni allusive filtrano poi dal colloquio tra i due moderni pellegrini e sarà proprio quella guida vestita di bianco, che dovrà condurre Pavese verso la cima, a rompere il «virtuoso» silenzio della campagna:

Mio cugino ha parlato stasera. Mi ha chiesto se salivo con lui: dalla vetta si scorge nelle notti serene il riflesso del faro.

«Perché non sali il dilettoso monte?» (Inf. I 77) diceva Virgilio, indicando a Dante la via per sfuggire le fiere che sbarravano l'accesso al colle della luce.

Quel gigante vestito di bianco che guidava Pavese era «tra i fortunati che han visto l'aurora / sulle isole più belle della terra», dove aleggia quel «dolce colore d'oriental zaffiro» che in Purgatorio accoglie le anime destinate alla salvezza. Anche lui, nel raccontare il suo viaggio agli antipodi, «adopera lento il dialetto, che, come le pietre / di questo stesso colle, è scabro tanto / che vent'anni di idiomi e di oceani diversi / non gliel'hanno scalfito», come aveva fatto l'avo Cacciaguida, che, pur avvolto dalla luce del mare paradisiaco, non ha dimenticato la lingua dell'infanzia e, «consolando, usava l'idioma / che prima i padri e le madri trastulla» (Par. XV 122-123).

Dall'intarsio di indizi appare evidente che Dante non viene rammemorato come un serbatoio di parole, ma è uno stato d'animo, il simbolo di una scrittura che «veste di corpo un concetto» (Pavese 2000: 163) e può agevolmente convivere con il sogno di un altro viaggiatore, che ha solcato gli oceani in cerca del mito. Separati anche graficamente, con la ripresa a margine e una pausa di silenzio aggiuntiva, due soli versi bastano a riportare il viaggio di Pavese da Dante a Melville: «Solo un sogno / gli è rimasto nel sangue: ha incrociato una volta / da fuochista su un legno olandese da pesca, il Cetaceo». 
Quando Pavese scrive i Mari del Sud, infatti, già si delineava nel suo orizzonte Moby Dick, ${ }^{8}$ che lui tradusse come un poema sacro cui non mancavano «né il cielo né la terra a por mano» e il mare era lo sbocco verso l'altrove, un varco per mondi diversi, ancora da leggere e da scrivere. Dopo i classici e Dante, assimilati alla scuola di Monti, era stato Melville a segnalargli la via per rappresentare per speculum la bassa realtà del paesaggio («la terra è bassa: lavorarla è fatica: curvi bisogna stare», secondo il detto materno $)^{9}$ e i vertici spirituali della letteratura (l'albero di trinchetto dove si rifugiava Ismaele per leggere Platone).

Le tappe del suo viaggio, insomma, sono costellate di citazioni latenti, libri che ha letto, eventi di parole che fanno parte di una biblioteca personale, naturale, dove anche il mare diventa una forma poetica che nulla toglie alle sue colline distese sull'orizzonte. Anzi, è proprio la campagna matrìa il luogo della «rivelazione», di straordinarie presenze, di effetti ottici che, come in un gioco di specchi, tengono insieme poesia, narrativa e autobiografia. «In principio è la Langa» recitava, infatti, l'incipit della biografia di Lorenzo Mondo, illuminando come un lampo non solo l'origine della creazione, ma un Verbo che può anche solcare il mare di Salgari tra le crete di Santo Stefano Belbo o incontrare tra flutti di colline il «gigante vestito di bianco» di un mitico Ulisse moderno tornato alla sua Itaca. Come Ulisse o Ismaele anche Pavese compie il suo viaggio, ma lo fa sulla carta, navigando a bordo di libri, senza andare lontano, prima giocando ai pirati malesi tra gli alberi, poi seduto in un cinema a Torino dove proiettavano film americani o in una piola di barriera che si chiamava Far West, ma anche ascoltando Monti che recitava versi come per un augurio «in nome di Dante». Il maestro si congedò dai suoi allievi dicendo che proprio nella lezione dantesca ciascuno di loro avrebbe potuto «vedere - cercare - trovare tutto quello che vorrà». Pavese rispose inviando una fotografia e un saluto «senza citazioni e senza frasi», perché - aggiungeva - «lei ci ha insegnato a porre ultima cosa nella vita i letterati. Le mostreremo la nostra riconoscenza con le nostre opere» (Monti 1965). Mantenne la parola, testimoniando che la «ricchezza di un'opera è sempre data dalla quantità di passato che contiene (Pavese 2000: 9, 344), da quel «magistero d'altri tempi di cui si conserva un distillato profumo», ora impresso in quel «niente che è tutto» che noi chiamiamo letteratura.

\section{Bibliografia}

ACCETTO, Torquato (1641): Della dissimulazione onesta, Napoli.

BECCARIA, Gian Luigi (1989): «...ma perché vengo da molto lontano: Cesare Pavese», in Le forme della lontananza. Milano, Garzanti, pp. 68-100.

BECCARIA Gian Luigi (2000): "Introduzione" a La luna e i falò. Torino, Einaudi, pp. v-xxx. DUGHERA, Attilio (1992): «Monti a Pavese: storia di un'amicizia attraverso le lettere», in Tra le carte di Pavese. Roma, Bulzoni, pp. 49-103.

\footnotetext{
${ }^{8}$ Nel gennaio del '31 Federico Gentile commissiona a Pavese la traduzione di Moby Dick che uscirà nel 1932.

9 Lo riporta anche una lettera di Monti del 5 novembre 1930.
} 
GuglielminetTi, Marziano (1990): Una poetica «tenzone»: Mario Sturani e Cesare Pavese, a cura di M. Mimita Lamberti. Torino, Allemandi.

LAJOLO, Davide (1960): Il «vizio assurdo». Milano, Il Saggiatore.

MESIANO, Luisella (2007): Cesare Pavese di carta e di parole. Bibliografia ragionata e analitica. Alessandria, Edizioni Dell'Orso.

MilA, Massimo (1977): «Prefazione» a Augusto Monti, Lettere a Luisotta. Torino, Einaudi.

Mondo, Lorenzo (2006): Quell'antico ragazzo. Vita di Cesare Pavese. Milano, Rizzoli.

MONTI, Augusto (1965): «Scuola di resistenza», in I miei conti con la scuola. Torino, Einaudi.

Mutterle, Anco M. (1977): L'immagine arguta. Lingua, stile, retorica di Pavese. Torino, Einaudi.

PAVESE, Cesare (1966a): Lettere I 1924-1944, vol. I, a cura di Lorenzo Mondo.Torino, Einaudi.

PAVESE, Cesare (1966b): Lettere II 1945-1950, vol. II, a cura di Italo Calvino.Torino, Einaudi.

PAVESE, Cesare (1974): Vita attraverso le lettere, a cura di Lorenzo Mondo. Torino, Einaudi.

PAVESE, Cesare (1989). Poesie giovanili (1923-1930), a cura di Attilio Dughera e Mariarosa Masoero. Torino, Einaudi (edizione fuori commercio).

PAVESE, Cesare (1990a): Pavese giovane. Torino, Einaudi.

PAVESE, Cesare (1990b): Mario Sturani: 1906-1978, a cura di M. Mimita Lamberti. Torino, Allemandi.

PAVESE, Cesare (1993): Lotte di giovani, a cura di Mariarosa Masoero. Torino, Einaudi.

PAVESE, Cesare (1998): Le poesie, a cura di Mariarosa Masoero, introduzione di M. Guglielminetti. Torino, Einaudi.

PAVESE, Cesare (2000a): Il mestiere di vivere. Diario 1935-1950, edizione condotta sull'autografo a cura di Marziano Guglielminetti e Laura Nay, introduzione di C. Segre. Torino, Einaudi.

PAVESE, Cesare (2000b): La luna e i falò. Torino, Einaudi. 